Chirurg 2009 $\cdot 80: 5-6$ DOI 10.1007/s00104-008-1654-z

Online publiziert: 16. Januar 2009

(c) Springer Medizin Verlag 2009

M.W. Büchler · M.N. Wente

Chirurgische Universitätsklinik, Klinikum der Universität Heidelberg

\title{
Funktions- und organerhaltende Pankreaschirurgie
}

In vorliegendem Heft von „Der Chirurg“ wird die Serie zur Pankreaschirurgie fortgesetzt.

Das Pankreaskarzinom hat nach wie vor eine schlechte Prognose, nicht hingegen die immer deutlicher charakterisierten Vorstufen. Die intraduktale papilläre muzinöse Neoplasie (IPMN) ist aktuell die an Häufigkeit im chirurgischen Krankengut am stärksten zunehmende Bauchspeicheldrüsenerkrankung weltweit [1]. Mittels moderner Diagnostik (CT/MRT) gelingt es zunehmend, die entweder vom Hauptgang (Main-duct-IPMN), vom Seitengang (Branch-duct-IPMN) oder als kombinierte Form von beiden Gangtypen ausgehende IPMN rechtzeitig zu diagnostizieren [2]. In der differenzierten chirurgischen Therapie dieser präkanzerösen Läsionen liegt eine große Chance, da dem Entstehen eines Pankreaskarzinoms zuvorgekommen werden kann. Der Artikel aus Heidelberg zum Thema IPMN beschreibt die wesentlichen Fortschritte der Bauchspeicheldrüsenchirurgie im Umgang mit diesem Tumor, weist jedoch auch auf die Einschränkungen der prognostischen Einschätzung aufgrund der noch nicht ausreichend wissenschaftlich erarbeiteten Evidenz hin.

\section{(2) Dass heute international zunehmend evidenzbasiert vorgegangen wird, ist das erfreuliche Ergebnis zahlreicher randomisiert kontrollierter Studien}

Die Etablierung funktions- und organerhaltender Pankreaschirurgie war das The- ma der vergangenen 15 Jahre. Die Artikel aus München (Friess) zur Segmentresektion, aus Heidelberg zur duodenumerhaltenden Pankreaskopfresektion und aus Hamburg (Izbicki) zur V-Shaped-Drainage des Pankreas erläutern den großen Stellenwert, den diese modernen Verfahren in der Zwischenzeit erreicht haben. Die chirurgische Therapie der chronischen Pankreatitis verlangt heute das gesamte Spektrum dieser funktions- und organerhaltenden Pankreaseingriffe, um den Leitsymptomen Schmerz, Pankreasgangobstruktion, Gallengangobstruktion bzw. entzündlicher Pankreaskopftumor individuell gerecht zu werden $[3,4]$. Eine der großen Innovationen des vergangenen Jahrhunderts, nämlich die duodenumerhaltende Pankreaskopfresektion nach Beger hat seit ihrer Erstbeschreibung im Jahre 1980 [5] mittlerweile einige klinisch wertvolle Modifikationen erfahren; zu nennen sind hier die Operation nach Frey [6], die Berner Modifikation [7] und die Hamburger Modifikation (V-Shaped) [8].

Dass beim Thema Pankreaschirurgie heute international zunehmend evidenzbasiert vorgegangen wird, ist das erfreuliche Ergebnis zahlreicher randomisiert kontrollierter Studien zum Stellenwert der organerhaltenden Verfahren, welche weit überwiegend in deutschen chirurgischen Kliniken durchgeführt wurden [9]. Auch hierin zeigen sich einmal mehr der Stellenwert klinischer Studien im Sinne der richtigen Anwendung moderner chirurgischer Verfahren und die Notwendigkeit, an multizentrischen Prüfungen teilzunehmen $[10,11]$. 
Wir danken den Autoren der zum Leitthema Pankreaschirurgie erstellten Artikel in den beiden aktuellen Ausgaben von „Der Chirurg“. Hierin wird deutlich dargestellt, wie rasch und von Innovationen geprägt sich die Bauchspeicheldrüsenchirurgie in den vergangenen 20 Jahren verändert hat.

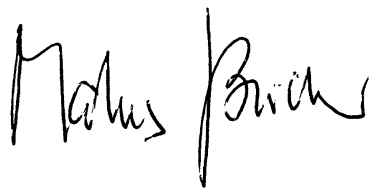

M. W. Büchler

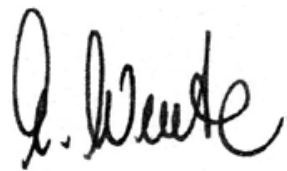

M. N. Wente

\section{Korrespondenzadresse}

\section{Prof. Dr. M.W. Büchler}

Chirurgische Universitätsklinik,

Klinikum der Universität Heidelberg,

Im Neuenheimer Feld 110,

69120 Heidelberg

Markus.Buechler@med.uni-heidelberg.de

\section{Literatur}

1. Belyaev O, Seelig MH, Muller CA et al (2008) Intraductal papillary mucinous neoplasms of the pancreas. J Clin Gastroenterol 42:284-294

2. Waters JA, Schmidt CM, Pinchot JW et al (2008) CT vs MRCP: optimal classification of IPMN type and extent. J Gastrointest Surg 12:101-109

3. Mihaljevic AL, Kleeff J, Friess H et al (2008) Surgical approaches to chronic pancreatitis. Best Pract Res Clin Gastroenterol 22:167-181

4. Büchler MW, Warshaw AL (2008) Resection versus drainage in treatment of chronic pancreatitis. Gastroenterology 134:1605-1607

5. Beger HG, Witte C, Krautzberger W, Bittner R (1980) Erfahrung mit einer das Duodenum erhaltenden Pankreaskopfresektion bei chronischer Pankreatitis. Chirurg 51:303-307

6. Frey CF, Smith GJ (1987) Description and rationale of a new operation for chronic pancreatitis. Pancreas 2:701-707

7. Gloor B, Friess H, Uhl W, Büchler MW (2001) A modified technique of the Beger and Frey procedure in patients with chronic pancreatitis. Dig Surg 18:21-25

8. Izbicki JR, Bloechle C, Broering DC et al (1998) Longitudinal V-shaped excision of the ventral pancreas for small duct disease in severe chronic pancreatitis: prospective evaluation of a new surgical procedure. Ann Surg 227:213-219

9. Diener MK, Rahbari NN, Fischer L et al (2008) Duodenum-preserving pancreatic head resection versus pancreatoduodenectomy for surgical treatment of chronic pancreatitis: a systematic review and meta-analysis. Ann Surg 247:950-961
10. Uhl W, Wente MN, Büchler MW (2000) Chirurgischklinische Studien in der praktischen Durchführung. Chirurg 71:615-625

11. McCulloch P, Taylor I, Sasako M et al (2002) Randomised trials in surgery: problems and possible solutions. BMJ 324:1448-1451
Liebe Leserinnen, liebe Leser,

"Der Chirurg" bietet Ihnen jeden Monat umfassende und aktuelle Beiträge zu interessanten Themenschwerpunkten aus allen Gebieten der operativen Medizin.

Möchten Sie ein bereits erschienenes Heft nachbestellen? Die folgenden Ausgaben können Sie direkt bei unserem Kundenservice zum Preis von je EUR 32,- beziehen:

\section{8}

- 1/2008 Chirurgie des Mediastinums

- 2/2008 Lebertransplantation

- 3/2008 Allgemeinchirurgie in der Diskussion

- 4/2008 Peritonitis

- 5/2008 Aktuelle Proktologie

- 6/2008 Die chronische Wunde

- 7/2008 Gastrointestinale Stromatumoren

- 8/2008 Benigne Lebertumoren inkl. Echinococcus

- 9/2008 Morbide Adipositas

- 10/2008 Wirbelsäulenchirurgie

- 11/2008 Hereditäre Karzinome

- 12/2008 Aktuelle Techniken in der Pankreaschirurgie - Teil 1

2009

- 1/2009 Aktuelle Techniken in der Pankreaschirurgie - Teil 2

- 2/2009 Neue Konzepte in der Endokrinen Viszeralchirurgie

- 3/2009 Weichteilsarkome

- 4/2009 Rektumkarzinom

- 5/2009 Diabetes mellitus

-6/2009 Thrombozytenaggregationshemmung

\section{So erreichen Sie unseren} Kundenservice:

Springer-Verlag

Kundenservice Zeitschriften

Haberstr. 7

69126 Heidelberg

Tel.: +49 $6221345-4303$

Fax: +49 $6221345-4229$

E-Mail: Leserservice@springer.com

\section{www.DerChirurg.de}

